\title{
Finite-difference methods for simulation models incorporating non-conservative forces
}

\author{
Keir E. Novik \\ Theory of Condensed Matter, Cavendish Laboratory, University of Cambridge, Madingley \\ Road, Cambridge, CB3 OHE, U.K. \\ ken21@cam.ac.uk \\ Peter V. Coveney \\ Schlumberger Cambridge Research, High Cross, Madingley Road, Cambridge, CB3 OEL, \\ U.K. \\ coveney@cambridge.scr.slb.com
}

(July 29, 1998)

\begin{abstract}
We discuss algorithms applicable to the numerical solution of second-order ordinary differential equations by finite-differences. We make particular reference to the solution of the dissipative particle dynamics fluid model, and present extensive results comparing one of the algorithms discussed with the standard method of solution. These results show the successful modeling of phase separation and surface tension in a binary immiscible fluid mixture.
\end{abstract}

\section{INTRODUCTION}

Computer simulations are a test-bed for theory, permitting experiments over which we have nearly complete control. The effectiveness of these computer experiments is limited by the power of available computers, and by their inherently discrete nature since the physical and chemical phenomena we study are continuous. Fortunately, mathematics has provided us with a framework into which we can place this problem: finite-differences and their continuous analogy, differential equations.

In the present paper, we shall be concerned with algorithms for solving secondorder ordinary differential equations of the form

$$
\ddot{x}=f(x, \dot{x}),
$$

where $x$ denotes a scalar or vector position, and dots indicate derivatives with respect to time. In particular, we are concerned with determining the motion of a system of particles moving according to the dissipative particle dynamics (DPD) computational fluid model (see Section III). The large number of equations and their non-linear nature make direct analytic mathematical solution infeasible, leaving numerical solution by finite-differences as the most appropriate approach. The forces in our computational fluid model are unusual in that they have a stochastic component, which depends on the size of the timestep in our finite-difference algorithm.

Traditional methods for solving Eq. (11) can be found in textbooks on numerical analysis, 26 and in papers and texts on molecular dynamics and related computational methods. 0 - 10 Algorithms from numerical analysis textbooks are usually unsuitable for our purpose because of the unstable nature of the motion of a manybody system, or an assumption that the forces do not depend on the timestep. Most of the other algorithms we find assume conservative forces, i.e. $\ddot{x}=f(x)$.

Throughout this paper, we use the notation $x_{n}=x(t)$ and $x_{n+1}=x(t+h)$ for the value of a variable (vector or scalar) at successive timesteps, where $h>0$ is the 
timestep width. Variables with tildes denote temporary quantities, vectors are in bold, and matrices are in a sans serif font.

\section{DISSIPATIVE PARTICLE DYNAMICS}

Hoogerbrugge and Koelman proposed DPD simulation of complex fluid behavior. This model is particularlyerell-suited to model multiphase flows, flowin porous media, colloidal suspensions, 11,12 microemulsions, and polymeric fluids. 13 The traditional approach of continuum fluid mechanics has met with limited success, and so many new micro- and mesoscopic approaches have been considered. In principle, molecular dynamics (MD) is the most accurate microscopic approach, although in practice it is too slow in both its quantum (CarParinello) and classical forms because of its excessive detail. Discrete methods developed from lattice-gas automata (LGA) have some success, but they too have problems, such as lacking Galilean invariance.14, 15

DPD was developed in an attempt to capture the best aspects of MD and LGA. It avoids the lattice-based problems of LGA, yet maintains an elegant simplicity and larger scale that keeps the model much faster than MD. This simplicity also makes DPD highly extensible, such as for including the interactions of complex molecules or modeling flow in an arbitrary number of spatial dimensions. The key features of the basic model are that the fluid is grouped into packets, termed "particles", and that mass and momentum are conserved but energy is not. Particle positions and momenta are real variables, and are not restricted to a grid.

There are two sequential steps to the action of the original model proposed by Hoogerbrugge and Koelman:U (i) an infinitesimally-short impulse step

$$
\mathbf{p}_{i, n+1}=\mathbf{p}_{i, n}+\sum_{j \neq i} \Omega_{i j} \hat{\mathbf{e}}_{i j}
$$

and (ii) a propagation step taking time $h$

$$
\mathbf{x}_{i, n+1}=\mathbf{x}_{i, n}+\frac{h}{m} \mathbf{p}_{i, n+1}
$$

where $\mathbf{p}_{i}$ and $\mathbf{x}_{i}$ are the momentum and position of particle $i, \hat{\mathbf{e}}_{i j}$ is the unit vector pointing from particle $j$ to particle $i, m$ is the mass of each particle, and

$$
\Omega_{i j}=\frac{3 m\left(1-\frac{r_{i j}}{r_{c}}\right)}{\pi r_{c}^{2} \rho}\left[\Pi_{i j}-\omega\left(\mathbf{p}_{i}-\mathbf{p}_{j}\right) \cdot \hat{\mathbf{e}}_{i j}\right]
$$

within the cut-off radius $r_{c}$. The quantity $r_{i j}$ denotes the distance separating particles $i$ and $j$, and $\rho$ is the mass density; note also that the normalization given is correct only in two dimensions. The random variable $\Pi_{i j}$ represents the conservative and stochastic effect of the collisions and gives rise to fluid pressure, while the second, dissipative, term inside the square brackets yields fluid viscosity.

Español and Warren's analysis 16 showed that the original DPD model does not satisfy detailed balance, so the equilibrium states (if they exist) cannot be simply characterized. Detailed balance is the condition equating the rates of forward and backward transition probabilities in a dynamical system, and is a sufficient (but not necessary) condition guaranteeing that the system has a (Gibbsian) equilibrium state 17.18 Español and Warren formulated a Fokker-Planck equation and equivalent set of stochastic differential equations which lead to a similar model, 


$$
\left\{\begin{aligned}
d \mathbf{p}_{i} & =\sum_{j \neq i} \mathbf{F}_{i j} d t=\sum_{j \neq i}\left[\mathbf{F}_{i j}^{C} d t+\mathbf{F}_{i j}^{D} d t+\mathbf{F}_{i j}^{R} d W_{i j}\right] \\
d \mathbf{x}_{i} & =\frac{\mathbf{p}_{i}}{m_{i}} d t .
\end{aligned}\right.
$$

In these equations, $\mathbf{p}_{i}, \mathbf{x}_{i}$, and $m_{i}$ denote the momentum, position, and mass of particle $i$, and $\mathbf{F}_{i j}^{C}$ is a conservative force acting between particles $i$ and $j$ while $\mathbf{F}_{i j}^{D}$ and $\mathbf{F}_{i j}^{R}$ are the dissipative and random forces; $d W_{i j}=d W_{j i}$ are independent increments of a Wiener process. By Itô calculus

$$
d W_{i j} d W_{k l}=\left(\delta_{i k} \delta_{j l}+\delta_{i l} \delta_{j k}\right) d t
$$

and so $d W_{i j}$ is an infinitesimal of order $\frac{1}{2}$ and we can write $d W_{i j}=\theta_{i j} \sqrt{d t}$, where $\theta_{i j}=\theta_{j i}$ is a random variable with zero mean and unit variance 17 With an appropriate choice for the form of the forces we find detailed balance is satisfied by this continuous-time version of DPD, 19 and so equilibrium states are guaranteed to exist and be Gibbsian. To ensure that the assqciated fluctuation-dissipation theorem holds, the forces assume the following forms: 16

$$
\begin{gathered}
\mathbf{F}_{i j}^{C}=\alpha \omega_{i j} \hat{\mathbf{e}}_{i j}, \\
\mathbf{F}_{i j}^{D}=-\gamma \omega_{i j}^{2}\left(\hat{\mathbf{e}}_{i j} \cdot \mathbf{v}_{i j}\right) \hat{\mathbf{e}}_{i j},
\end{gathered}
$$

and

$$
\mathbf{F}_{i j}^{R}=\sigma \omega_{i j} \hat{\mathbf{e}}_{i j},
$$

where $\mathbf{v}_{i j}=\mathbf{p}_{i} / m_{i}-\mathbf{p}_{j} / m_{j}$ is the difference in velocities of particles $j$ and $i, \hat{\mathbf{e}}_{i j}$ is the unit vector pointing from particle $j$ to particle $i$, and $\omega_{i j}$ is a weighting function depending only on the distance separating particles $i$ and $j$. The constants $\alpha, \gamma$, and $\sigma$ are chosen to reflect the relative importance of the conservative, dissipative (viscous), and random components in the fluid of interest. As a consequence of detailed balance and the fluctuation-dissipation theorem, $\gamma$ and $\sigma$ are related to Boltzmann's constant $k_{B}$ and the equilibrium temperature $T$ by

$$
\frac{\sigma^{2}}{\gamma}=2 k_{B} T
$$

In order to remain as close as possible to the original DPD model, we choose the friction weight function to be

$$
\omega_{i j}=1-\frac{r_{i j}}{r_{c}}
$$

within the constant cutoff length $r_{c}>0$, where $r_{i j}$ is the distance between particles $i$ and $j$. Adding Eqs. (8)-(9), the total force is

$$
\mathbf{F}_{i j}=\left[\alpha-\gamma \omega_{i j}\left(\hat{\mathbf{e}}_{i j} \cdot \mathbf{v}_{i j}\right)+\frac{\sigma \theta_{i j}}{\sqrt{d t}}\right] \omega_{i j} \hat{\mathbf{e}}_{i j} .
$$

In summary, the main changes from the original DPD model are the specification of the motion in terms of differential equations instead of an impulse step followed by coasting; the separation of the conservative, dissipative, and random forces, with the strength of each being controlled by the new constants $\alpha, \gamma$, and $\sigma$; the insertion of an extra factor of $\omega_{i j}$ in front of the dissipative force; the specification of 
the thermodynamic temperature in terms of the model parameters; and the square root dependence of the random force on size of timestep.

In order to model binary immiscible fluids, we adopt the simplest approach of introducing a new variable, the "color", by analogy with Rothman-Keller 20 When two particles of different color interact we increase the conservative force, thereby increasing the repulsion. That is,

$$
\alpha \mapsto \alpha_{i j}= \begin{cases}\alpha_{0} & \text { if particles } i \text { and } j \text { are the same color } \\ \alpha_{1} & \text { if particles } i \text { and } j \text { are different colors }\end{cases}
$$

where $\alpha_{0}$ and $\alpha_{1}$ are constants with $0 \leq \alpha_{0}<\alpha_{1}$. As for the single-phase DPD fluid, the Navier-Stokes equations are obeyed within regions of homogeneity in each of the two immiscible fluids, while detailed balance is preserved, at least in the limit of continuous time.21 We would like to emphasize that with the sole exception of the choice of finite-difference algorithm, this model is identical to that used in our most-recently published simulations 22

\section{EVALUATION CRITERIA}

A good finite-difference method should be fast, stable, accurate, easy to implement, and require little storage. Since for most large systems the force evaluation dominates the computation time, the fastest algorithm is the one that evaluates the force the fewest times for a given accuracy. In general, this means that stable methods which allow large time steps are preferable. In this context we must be careful to distinguish the stability of a method from the stability of the system being studied, as the dynamical systems we wish to study are extremely unstable, with small perturbations growing exponentially 6.23 A stable method will respond better to large sizes of timestep than an unstable method. The accuracy of an algorithm is difficult to measure in practice because of the extreme instability of our dynamical systems. Practical accuracy is best quantified by requiring a method to give a trajectory in phase space that is physical for the system (i.e. similar to that observed in experiments), and for which calculated properties of the system are close to their theoretical values. An idea of the accuracy of a method can also be obtained by considering the local truncation error, expressed in terms of the order of the timestep occurring in the first truncated term of the corresponding Taylor series. Because the forces considered are not smooth, low order algorithms may possibly be more accurate than high order algorithms.

What sriteria can we use to evaluate the suitability of a method? The literature 9.23 claims that a good finite-difference method will be area preserving, time reversible, energy conserving, and ergodic. The first three criteria are closely related. We need to be careful in distinguishing between the properties of the finitedifference algorithm and those of the system it is solving. For example, when we describe an algorithm as being time reversible, we are not saying that it corrects or compensates for the lack of time symmetry in a non-conservative system, but rather that when applied to a conservative system this finite-difference algorithm is able to retrace the precise sequence of steps in the backward evolution which it traversed originally in the forward time direction. A similar confusion may arise when we consider ergodicity. Is this not a property normally associated with the rules of interaction of systems? What does it mean to say that a finite-difference algorithm is ergodic? When we describe an algorithm as being ergodic, we mean that an ergodic system updated by this algorithm will still sample the full phase space; a non-ergodic algorithm updating the same system will not. 
We also need to consider the relevance of these four criteria to non-conservative forces. For example, is there any use in having an algorithm which conserves energy when our DPD interactions conserve only momentum? We argue that unnecessary losses are indeed undesirable; a finite-difference algorithm which loses energy by consistently underestimating velocity would exhibit lower temperature than a similar algorithm which does not.

An algorithm which preserves the volume of phase space will have a unit Jacobian for the transformation of variables from one timestep to the next. Time reversibility can also be checked analytically (ignoring for the moment the properties of the forces). Energy conservation normally follows from time reversibility, but it can also be verified for a conservative system, such as the (one-dimensional) simple harmonic oscillator

$$
\ddot{x}=-x,
$$

where the total energy is $E=\left(x^{2}+\dot{x}^{2}\right) / 2$.

Ergodicity can be checked by calculating the equilibrium temperature and pressure of an homogeneous fuid, and comparing the velocity distribution with a normal (Gaussian) distribution. 9 As this is not a sufficient condition, in the present paper we also simulate a binary immiscible fluid using each of the algorithms, testing Laplace's law and comparing the rate of growth of domains with the extensive experimental and theoretical literature, and with our earlier simulations22 24 using the standard DPD finite-difference algorithm (see Section IV A). It has also been noted in DPD simulations of an ideal gas (i.e. $\alpha=0$ ) using this alorithm that the radial pair-correlation function differs from the theoretical result 25 A good finitedifference algorithm would remove this inconsistency, so we verify this as well.

\section{ALGORITHMS CONSIDERED FOR CONTINUOUS-TIME DPD}

For molecular dynamics simulations, the literaturerecommends either one of the Verlet schemes or a multi-value predictor-corrector 6.9 . 23 Runge-Kutta and extrapolation methods are considered unsuitable because they typically require a large number of force evaluations per timestep, which is inefficient for a many-particle system. For the same reason, methods which consider the second order differential equation directly are usually preferred to methods which break it into two first order equations.

We do not consider implicit methods (in which a variable appears on both sides of a single equation) since they are computationally infeasible for any system large enough to be of interest. This is so because the simplest implicit method for a system of $N$ particles requires solution of $N$ interdependent linear equations taking $\mathrm{O}\left(N^{3}\right)$ operations 1 compared with the $\mathrm{O}(N)$ operations of an explicit method. The need to solve these interdependent equations also makes implicit methods much more complicated than explicit methods. However, where the differential equations are very stiff (have several greatly differing time scales) the greatly-increased algorithmic stability could outweigh the disadvantages. Having had reasonable results with explicit methods, we do not believe our system is so stiff as to require implicit schemes.

We have obtained good results by starting our simulations with all particles at rest, with the initial position of each particle typically selected from a random variable uniformly distributed across the simulation space. Particles are assumed to have been at rest for some time, so prior positions are the same as initial positions, and prior velocities and higher derivatives are all zero. In our notation $x_{n}=x(t)$ and $x_{n+1}=x(t+h)$, the initial conditions at $t=0$ correspond to $n=0$ while 
negative values of $n$ represent values prior to the start of the simulation. To give an idea of the relative accuracy of each algorithm we report the local truncation error in position as the order of the timestep appearing in the first term omitted from the Taylor series.

\section{A. Basic methods}

The original algorithm proposed by Hoogerbrugge and Koelmant is a modified Euler scheme

$$
\begin{aligned}
\ddot{x}_{n} & =f\left(x_{n}, \dot{x}_{n}, h\right) \\
\dot{x}_{n+1} & =\dot{x}_{n}+h \ddot{x}_{n} \\
x_{n+1} & =x_{n}+h \dot{x}_{n+1} \\
& =x_{n}+h\left(\dot{x}_{n}+h \ddot{x}_{n}\right) .
\end{aligned}
$$

As much researeh been published using this area-preserving scheme, 1, 13,13,22.24 26 it is important to compare it with the other methods. The local truncation error is $\mathrm{O}\left(h^{2}\right)$.

\section{B. Verlet-based methods}

Due to the velocity-dependent nature of the force (Eq. (12)), the traditional $\mathrm{O}\left(h^{3}\right)$ time-reversible velocity-Verlet algorithm

$$
\begin{aligned}
& x_{n+1}=x_{n}+h\left(\dot{x}_{n}+\frac{h}{2} \ddot{x}_{n}\right) \\
& \ddot{x}_{n+1}=f\left(x_{n+1}\right) \\
& \dot{x}_{n+1}=\dot{x}_{n}+\frac{h}{2}\left(\ddot{x}_{n}+\ddot{x}_{n+1}\right)
\end{aligned}
$$

cannot be used. Ferrario 3 has suggested two modified schemes to get around this problem. The first is

$$
\begin{aligned}
\ddot{x}_{n} & =f\left(x_{n}, \dot{x}_{n}, h\right) \\
x_{n+1} & =2 x_{n}-x_{n-1}+h^{2} \ddot{x}_{n} \\
\dot{x}_{n} & =\frac{x_{n+1}-x_{n-1}}{2 h} \\
\dot{x}_{n+1} & =\dot{x}_{n-1}+2 h \ddot{x}_{n}
\end{aligned}
$$

and the second

$$
\begin{aligned}
x_{n+1} & =x_{n}+h\left(\dot{x}_{n}+\frac{h}{2} \ddot{x}_{n}\right) \\
\tilde{\dot{x}} & =\dot{x}_{n-1}+2 h \ddot{x}_{n} \\
\ddot{x}_{n+1} & =f\left(x_{n+1}, \tilde{\dot{x}}, h\right) \\
\dot{x}_{n+1} & =\dot{x}_{n}+\frac{h}{2}\left(\ddot{x}_{n}+\ddot{x}_{n+1}\right) .
\end{aligned}
$$

These two $\mathrm{O}\left(h^{3}\right)$ methods are hereafter referred to as Ferrario I (Eq. (17)) and Ferrario II (Eq. 18) respectively. Groot and Warren 8 recently proposed a different velocity estimate for use with the velocity-Verlet algorithm. Their method is

$$
x_{n+1}=x_{n}+h\left(\dot{x}_{n}+\frac{h}{2} \ddot{x}_{n}\right)
$$




$$
\begin{aligned}
\tilde{\dot{x}} & =\dot{x}_{n}+\lambda h \ddot{x}_{n} \\
\ddot{x}_{n+1} & =f\left(x_{n+1}, \tilde{\dot{x}}, h\right) \\
\dot{x}_{n+1} & =\dot{x}_{n}+\frac{h}{2}\left(\ddot{x}_{n}+\ddot{x}_{n+1}\right) .
\end{aligned}
$$

This is conceptually similar to the leap-frog form of the Verlet algorithm, for if $\lambda \neq 1$ the force is evaluated when the positions and velocities are out of phase. A reasonable range for $\lambda$ would be from 0 to 1 ; we consider only $\lambda=\frac{1}{2}$ and $\lambda=1$. For conservative forces Eq. (19) reduces to Eq. (16); otherwise with $\lambda=1$ its local truncation error is $\mathrm{O}\left(h^{3}\right)$, and $\mathrm{O}\left(h^{2}\right)$ for $\lambda \neq 1$.

\section{Runge-Kutta methods}

In order to confirm the poor performance of Runge-Kutta schemes, we consider a two-stage explicit Runge-Kutta method of the form proposed by Gear $3^{3}$ for the direct solution of second order differential equations:

$$
\begin{aligned}
\tilde{f}_{1} & =f\left(x_{n}, \dot{x}_{n}, \frac{2}{3} h\right) \\
\tilde{x} & =x_{n}+\frac{2}{3} h\left(\dot{x}_{n}+\frac{h}{3} \tilde{f}_{1}\right) \\
\tilde{\dot{x}} & =\dot{x}_{n}+\frac{2}{3} h \tilde{f}_{1} \\
\tilde{f}_{2} & =f\left(\tilde{x}, \tilde{\dot{x}}, \frac{1}{3} h\right) \\
x_{n+1} & =x_{n}+h\left(\dot{x}_{n}+\frac{h}{4}\left(\tilde{f}_{1}+\tilde{f}_{2}\right)\right) \\
\dot{x}_{n+1} & =\dot{x}_{n}+\frac{h}{4}\left(\tilde{f}_{1}+3 \tilde{f}_{2}\right) .
\end{aligned}
$$

In applying this $\mathrm{O}\left(h^{4}\right)$ method to our system, we must be careful to choose the appropriate length of timestep for each force evaluation.

\section{Multi-value predictor-correctors}

Gear has proposed a notation for the general class of multi-value predictorcorrector algorithms. 3 The variables to be stored from step to step are kept in a column vector, usually in either the Nordsieck $(N)$ representation

$$
\mathbf{y}_{n}(N)=\left(x_{n}, h \dot{x}_{n}, \frac{h^{2}}{2} \ddot{x}_{n}, \frac{h^{3}}{3 !} x_{n}^{(i i i)}, \frac{h^{4}}{4 !} x_{n}^{(i v)}, \ldots\right)^{T}
$$

or the force $(F)$ representation

$$
\mathbf{y}_{n}(F)=\left(x_{n}, h \dot{x}_{n}, \frac{h^{2}}{2} \ddot{x}_{n}, \frac{h^{2}}{2} \ddot{x}_{n-1}, \frac{h^{2}}{2} \ddot{x}_{n-2}, \ldots\right)^{T} .
$$

The $N$-representation is more convenient for changing timestep and order, while the $F$-representation simplifies calculation. Transformation from one representation to the other is straightforward, involving a multiplication by a transformation matrix T. Gear claims that transformation from one representation to another does not 
affect the truncation error or stability of the method, but does affect the round-off error, and amount of computation and storage 3.2

To take a step forward in time using a predictor-corrector method, we first multiply the column vector $\mathbf{y}_{n-1}$ with a matrix $\mathrm{A}$ in an explicit prediction step

$$
P: \quad \mathbf{y}_{n, 0}=\mathrm{Ay}_{n-1}
$$

and then the forces are evaluated at the positions and velocities in this vector,

$$
E_{m}: \quad \ddot{x}_{n,(m+1)}=f\left(x_{n, m}, \dot{x}_{n, m}, h\right) .
$$

A corrected vector is obtained by adding a multiple of the difference between the predicted and the calculated force to the existing vector

$$
C_{m}: \quad \mathbf{y}_{n,(m+1)}=\mathbf{y}_{n, m}+\mathbf{l} \frac{h^{2}}{2}\left(\ddot{x}_{n,(m+1)}-\ddot{x}_{n, m}\right) .
$$

It is possible to iterate the force evaluation $(E)$ and correction $(C)$ steps after the initial prediction $(P)$, leading to a $P(E C)^{q}$ or $P(E C)^{q} E$ algorithm. The subscript $m$ denotes each evaluation-correction step from the first $(m=0)$ through the last $(m=q-1$, i.e. $0 \leq m<q)$. It can be shown that in the limit of a large number of iterations $(q \gg 1)$, these algorithms are time-reversible 9

The predictor matrix A is usually chosen to be the Pascal triangle matrix

$$
\mathrm{A}(N)=\left(\begin{array}{lllll}
1 & 1 & 1 & 1 & \\
0 & 1 & 2 & 3 & \\
0 & 0 & 1 & 3 & \\
0 & 0 & 0 & 1 & \\
& & & & \ddots
\end{array}\right)
$$

(in the $N$-representation), which means that Eq. (23) predicts according to the familiar Taylor series expansion. The column vector 1 is chosen by accuracy and stability arguments. The corrector vectors for 3 - and 4 -value method\$28 to solve second order differential equations in the $N$-representation are

$$
\mathbf{l}(N)=\left(\frac{1}{3}, 1,1\right)^{T}
$$

and

$$
\mathbf{l}(N)=\left(\frac{1}{6}, \frac{5}{6}, 1, \frac{1}{3}\right)^{T}
$$

giving local truncation error of $\mathrm{O}\left(h^{4}\right)$ and $\mathrm{O}\left(h^{5}\right)$ respectively. The correction vectors are identifal in the $F$-representation except that all components beyond the third are zero. 3 U

We would like to consider these two methods further modified by the insertion of a factor $\lambda$ (typically ranging from 0 to 1 ) into the velocity prediction:

$$
\mathrm{A}(N)=\left(\begin{array}{cccc}
1 & 1 & 1 & 1 \\
0 & 1 & 2 \lambda & 3 \\
0 & 0 & 1 & 3 \\
0 & 0 & 0 & 1
\end{array}\right),
$$

(this matrix being for the 4 -value method). While this predicted value of velocity will be used for the first force evaluation, the Taylor-predicted velocity will be used 
in the correction step and subsequent iterations so that the methods will benefit from the modified velocity estimate and yet will still converge to the correct solution. It can be shown that both Eqs. (26) and (29) are area-preserving transformations. Written explicitly in the $P(E C)^{q} F$-representation, we have

$$
\begin{gathered}
P:\left\{\begin{array}{l}
x_{n, 0}=x_{n-1}+h\left(\dot{x}_{n-1}+\frac{h}{2} \ddot{x}_{n-1}\right) \\
\dot{x}_{n, 0}=\dot{x}_{n-1}+h \ddot{x}_{n-1} \\
\ddot{x}_{n, 0}=\ddot{x}_{n-1}
\end{array}\right. \\
E_{m}: \quad \ddot{x}_{n,(m+1)}= \begin{cases}f\left(x_{n, 0}, \dot{x}_{n-1}+\lambda h \ddot{x}_{n-1}, h\right) & \text { if } m=0 \\
f\left(x_{n, m}, \dot{x}_{n, m}, h\right) & \text { if } m>0\end{cases} \\
C_{m}:\left\{\begin{array}{l}
x_{n,(m+1)}=x_{n, m}+\frac{h^{2}}{6}\left(\ddot{x}_{n,(m+1)}-\ddot{x}_{n, m}\right) \\
\dot{x}_{n,(m+1)}=\dot{x}_{n, m}+\frac{h}{2}\left(\ddot{x}_{n,(m+1)}-\ddot{x}_{n, m}\right)
\end{array}\right.
\end{gathered}
$$

and

$$
\begin{gathered}
P:\left\{\begin{array}{c}
x_{n, 0}=x_{n-1}+h\left(\dot{x}_{n-1}+\frac{h}{6}\left(4 \ddot{x}_{n-1}-\ddot{x}_{n-2}\right)\right) \\
\dot{x}_{n, 0}=\dot{x}_{n-1}+\frac{h}{2}\left(3 \ddot{x}_{n-1}-\ddot{x}_{n-2}\right) \\
\ddot{x}_{n, 0}=2 \ddot{x}_{n-1}-\ddot{x}_{n-2} \\
\ddot{x}_{(n-1), 0}=\ddot{x}_{n-1}
\end{array}\right. \\
E_{m}: \ddot{x}_{n,(m+1)}=\left\{\begin{array}{l}
f\left(x_{n, 0}, \dot{x}_{n-1}+\lambda \frac{h}{2}\left(3 \ddot{x}_{n-1}-\ddot{x}_{n-2}\right), h\right) \\
f\left(x_{n, m}, \dot{x}_{n, m}, h\right)
\end{array}\right. \\
C_{m}:\left\{\begin{array}{l}
\text { if } m=0 \\
x_{n,(m+1)}=x_{n, m}+\frac{h^{2}}{12}\left(\ddot{x}_{n,(m+1)}-\ddot{x}_{n, m}\right) \\
\dot{x}_{n,(m+1)}=\dot{x}_{n, m}+\frac{5}{12} h\left(\ddot{x}_{n,(m+1)}-\ddot{x}_{n, m}\right) \\
\ddot{x}_{(n-1), 0}=\ddot{x}_{(n-1), m}
\end{array}\right.
\end{gathered}
$$

for the 3- and 4-value methods respectively.

\section{PERFORMANCE OF THE ALGORITHMS}

In this section we report on our evaluation of the finite difference algorithms in the previous section, our evaluation criteria being briefly outlined in Section III. We first present the results of the simple evaluations checking conservation of energy and ergodicity, before presenting more extensive results on phase separation and surface tension. 


\section{A. Energy conservation}

A system consisting of a single simple harmonic oscillator was allowed to evolve for a thousand timesteps from an initial configuration with unit position $(x=1)$. Two sizes of timestep were considered: one tenth and one hundredth the period of the oscillator. The variation of the energy $E=\left(x^{2}+\dot{x}^{2}\right) / 2$ relative to the initial energy $E=\frac{1}{2}$ for each of these sets of simulations is shown in Tables [I and II] respectively; results are identical for $\lambda=\frac{1}{2}$ and $\lambda=1$. Where a median and standard deviation are given, the energy varies periodically; where an exponent is given, the energy grows or decays as $a \exp (b t)$. From these tables we can see that Gear-3 $(q=1)$ and Runge-Kutta have an undesirable continual energy loss for both sizes of timestep. The other Gear methods show a periodic variation of energy for the small timestep, but display a long-term energy drift for the large timestep. Both Ferrario methods, Groot-Warren, and Hoogerbrugge-Koelman only show periodic variation in energy, with Hoogerbrugge-Koelman showing by far the largest magnitude of variation. However, none of the other methods include the correct (unit) relative energy within the range of their variation, and so we cannot conclude that one periodically varying method is significantly better than any other.

\section{B. Ergodicity}

Two-dimensional DPD systems of 1600 identical particles were allowed to evolve to equilibrium (roughly $t=50$ ), and then the temperature $k_{B} T$ and pressure $P$ were observed for the same length of time again. This was repeated three times for each situation from different random initial configurations, and the results averaged. The exact parameters used in these simulations are shown in Table III. The units of these parameters are those natural to the DPD simulation; although no one has yet related them to physical scales of length or time, it is possible to do so if one desires to apply the simulation technique to describe a real fluid. These particular values were suggested by Hoogerbrugge and Koelman.

From statistical mechanics, we know that the instantaneous pressure $(P)$ of a system in terms of the internal virial $(W)$ and the instantaneous temperature $(T)$ are given by

$$
P=\frac{N k_{B} T+W}{V}
$$

where

$$
N k_{B} T=\frac{1}{2} \sum_{i} m_{i}\left|\dot{\mathbf{x}}_{i}\right|^{2}
$$

and

$$
W=\frac{1}{2} \sum_{i} \sum_{j>i} \mathbf{r}_{i j} \cdot \mathbf{F}_{i j}
$$

where $V$ is the physical volume of space, $\mathbf{r}_{i j}=\mathbf{x}_{i}-\mathbf{x}_{j}$, and $|\cdot|$ indicates vector magnitude. The thermodynamic pressure and temperature are the time averages of the instantaneous quantities. Because of the work of Español, Warren, and Coveney 1621 we can be sure that a temperature exists and is meaningful, at least in the continuous-time limit. The pressure was calculated immediately after the final force evaluation in each algorithm; the temperature was further evaluated using the 
final values of velocity. At the end of each simulation, the velocity distribution in the $x$-direction was examined; it was statistically indistinguishable from the Maxwellian distribution whenever the temperature converged.

With the exception of Runge-Kutta, all the methods converge to correct temperature and pressure as we decrease the timestep. We believe this error is due to the unusual timestep-dependent nature of the force, because the Runge-Kutta algorithm divides the motion into two steps of two-thirds and then one-third the total timestep. Of all the algorithms we consider, only the Runge-Kutta scheme subdivides the timestep. The relative proportion of the random component of the force changes when we reduce the timestep size, and the algorithm does not properly take this into account.

As we can see in Figs. 1 and 2 (in which $k_{B} T$ is plotted relative to its theoretical value), the Groot-Warren $\left(\lambda=\frac{1}{2}\right)$ and Gear-3 $\left(\lambda=\frac{1}{2}, q=1\right)$ methods give the most accurate temperature. Figs. 3 and 1 show that the pressure (in DPD units) is most accurate for a given timestep with the Gear-3 $(\lambda=1, q=2)$ and Gear-4 $\left(\lambda=\frac{1}{2}\right.$ and $\lambda=1, q=2)$ methods, with Gear-3 $\left(\lambda=\frac{1}{2}, q=1\right.$ and $\left.q=2\right)$, Groot-Warren $\left(\lambda=\frac{1}{2}\right)$, and Hoogerbrugge-Koelman following close behind. (Groot-Warren $(\lambda=$ $\frac{1}{2}$ ) and Hoogerbrugge-Koelman gave nearly identical results for pressure, so their symbols are superimposed in Figs. 3 and 4 -look under the curve with the filled squares: $\operatorname{Gear}-3\left(\lambda=\frac{1}{2}, q=1\right)$.) The algorithms are unstable for timesteps larger than those shown in Figs. 1 and 3 .

Excluding the time taken to calculate the temperature and pressure, these simulations take $140 \mathrm{~ms}$ per timestep for the Hoogerbrugge-Koelman algorithm on a $133 \mathrm{MHz}$ DEC Alpha. Because of the large number of particles involved, the speed of the algorithms is almost directly proportional to the number of force evaluations per timestep: one for Hoogerbrugge-Koelman, the Ferrario methods, and GrootWarren; two for Runge-Kutta; and $q$ for the Gear predictor-correctors.

\section{Phase separation kinetics}

The study of growth kinetics in binpry immiscible fluids has received much attention lately (see our previous papers2224 for detailed references, but note also some more recent work30.11). A central quantity is the characteristic domain size $R(t)$; typically, one finds that

$$
R(t) \sim t^{\beta} .
$$

Without hydrodynamic interactions, theory and experiment 29 tell us that the scaling exponent $\beta=\frac{1}{3}$. If flow effects are relevant

$$
\begin{aligned}
& \beta=\left\{\begin{array}{ll}
\frac{1}{2} & \text { for } R \ll R_{h} \\
\frac{2}{3} & \text { for } R \gg R_{h}
\end{array} \quad\right. \text { (in two dimensions) } \\
& \beta=\left\{\begin{array}{ll}
\frac{1}{3} & \text { for early-time } R \ll R_{d} \\
1 & \text { for late-time } R_{d} \ll R \ll R_{h} \\
\frac{2}{3} & \text { for } R \gg R_{h}
\end{array} \quad\right. \text { (in three dimensions), }
\end{aligned}
$$

where $R_{h}=\eta^{2} /(\rho \tau)$ is the hydrodynamic length and $R_{d}=\sqrt{\eta D}$ is the diffusive length, expressed in terms of the absolute (a.k.a. dynamic) viscosity $\eta$ (dimensional analysis suggests it is not the kinematic viscosity $\nu=\eta / \rho$, as previously thought22 24), density $\rho$, surface tension $\tau$, and diffusion coefficient $D$. 
Because of the compute-intensive nature of the simulations in this and the following section, we had time enough on to use a single finite-difference method. Our previously performed simulations22 24 used the Hoogerbrugge-Koelman algorithm, and of all the methods Groot-Warren $\left(\lambda=\frac{1}{2}\right)$ and Gear-3 $\left(\lambda=\frac{1}{2}, q=1\right)$ gave the most accurate temperature and pressure. We chose to use the Groot-Warren algorithm over Gear-3 because of its superior energy conservation.

Two dimensional systems of 40000 particles were allowed to evolve from a symmetric quench, the initial random configuration differing for each of several simulations. The model parameters are identical to those used for the smaller simulations (see Table III). Observing the growth of those simulations which used the Groot-Warren algorithm $\left(\lambda=\frac{1}{2}, h=0.1\right)$ indicated two regimes of growth following Eq. (35): $\beta=\frac{1}{2}(0.478 \pm 0.004)$ crossing over to $\beta=\frac{2}{3}(0.65 \pm 0.02)$ at $R=8.7 \pm 0.4$. Reducing the timestep to $h=0.05$, we observe nearly identical results: $\beta=\frac{1}{2}(0.476 \pm 0.004)$ crossing to $\beta=\frac{2}{3}(0.63 \pm 0.03)$ at $R=7.4 \pm 0.9$. Error ranges given are the $68 \%$ confidence intervals for the mean, using approximately five simulations for each configuration. Phase separation did not occur in symmetric quenches with a larger timestep $(h=0.5)$ for which the algorithm is unstable. This is not surprising, as the temperature is undefined and diverges to infinity, so that the system does not remain quenched. At this size of timestep, the fluid modeled approximates a gas, which we expect to remain in a thoroughly mixed state.

Asymmetric quenches with a 60:40 majority:minority phase (color) ratio (GrootWarren $\left.\lambda=\frac{1}{2}, h=0.1\right)$ displayed growth with $\beta=\frac{1}{2}(0.476 \pm 0.002$ crossing to $0.547 \pm 0.003$ at $R=7.4 \pm 0.7$ ). Asymmetric quenches with a 70:30 ratio (GrootWarren $\left.\lambda=\frac{1}{2}, h=0.1\right)$ also gave $\beta=\frac{1}{2}(0.460 \pm 0.003)$. Special simulations were also set up, in which the conservation of momentum was violated by $10-15 \%$ for each interaction (Groot-Warren $\lambda=\frac{1}{2}, h=0.1$ ) by adding a random vector to the velocity of each particle every timestep. These simulations demonstrated a growth exponent of $\beta=0.407 \pm 0.006$, ceasing growth entirely at $R=7.57 \pm 0.05$. This suggests that momentum conservation is necessary for the viscous mechanism of phase separation. The observed growth exponent is close to the theoreticallypredicted $\beta=\frac{1}{3}$.

A sample log-log plot of the characteristic domain size $(R)$ against time for a single symmetric quench (Groot-Warren $\lambda=\frac{1}{2}, h=0.1$ ) is shown in Fig. 5 . In this figure we can see the initial non-algebraic growth as the system settles down from its quench, the $\beta=\frac{1}{2}$ early-time diffusive scaling regime, and the $\beta=\frac{2}{3}$ late-time viscous scaling regime. The results presented in this section are qualitatively the same as these-pbtained in our earlier simulations using the Hoogerbrugge-Koelman algorithm.22 24

\section{Domain surface tension}

Phase separation in binary fluids depends, among other things, on the interfacial tension which exists between the two immiscible phases. A further important test of our algorithms is thus to check on the existence of a surface tension by confirming the validity of Laplace's law using a series of bubble simulations.22 24

Our procedure is to set up a circular bubble (radius $R$ ) of one color phase within the other phase, and allow the bubble to reach equilibrium. We then calculate the pressure inside $(r<0.7 R)$ and outside $(r>1.3 R)$ of the bubble, using Eqs. (32 34). Repeating these experiments for various size bubbles, we can verify Laplace's law

$$
P_{\text {in }}-P_{\text {out }}=\frac{\tau}{R}
$$


From our results in Fig. 6 (Groot-Warren, $\lambda=\frac{1}{2}, h=0.1$ ) we can see that we have the desired linear behavior, and estimate $\tau$, the interfacial surface tension, to be $0.33 \pm 0.05$ This is larger than observed in our earlier Hoogerbrugge-Koelman simulations, 224 but because of the greatly decreased noise with the Groot-Warren $\left(\lambda=\frac{1}{2}\right)$ method, we are more confident in the accuracy of the current results.

\section{E. Viscosity}

The absolute (dynamic) viscosity of this system can be estimated theoretically from the continuous-time viscosity 25 as $\eta=2.8 \pm 0.4$, where the error is assumed to be of the same order of magnitude as that of the temperature. In order to verify this estimate, we performed a series of simulations of steady shear of an homogeneous fluid, using Lees-Edwards periodic boundary conditions. Because we intend to use this value of viscosity to determine the hydrodynamic length $R_{h}$ for comparison with the spinodal decomposition simulations in Section $\mathrm{VC}$, we continue to use only the Groot-Warren algorithm $\left(\lambda=\frac{1}{2}\right)$. A total of 63 simulations were performed, each from a different random initial configuration. Systems of both 1600 and 6400 particles were studied, six simulations at each of nine different shear rates for the former and three simulations at each of three distinct shear rates for the latter. As the results from the larger simulations gave a mean viscosity nearly identical to that of the smaller ones, we can conclude that domain size effects do not bias the smaller, faster simulations. The velocity profile was calculated for each set of parameters, and was found to be statistically indistinguishable from linear in every case.

Analyzing these simulations led to a conclusion of $\eta=1.94 \pm 0.01$. Fig. 7 displays the results; the error bars are the $68 \%$ confidence intervals for the mean, equally weighting each of the simulations for each set of parameters. Others have also found discrepancies between thegry and simulation, particularly regarding the kinematic contribution to viscosity 25

Using the surface tension and viscosity calculated from our simulations, we can estimate the hydrodynamic length $R_{h}=\eta^{2} /(\rho \tau)$ to be $2.9 \pm 0.5$. During our simulations we observed the typical domain size of the crossover in spinodal decomposition, which we estimate as $8 \pm 1$ (see Section $\mathrm{VG}$ ). This length is in agreement with Eq. (36), since for length scales much less than $R_{h}$ we typically see a growth exponent of $\beta=\frac{1}{3}$, while for length scales much larger than $R_{h}$ we typically see $\beta=\frac{2}{3}$. Although it would be more reassuring if $R_{h}$ and our observed crossover length were more comparable in size, we must remember that the theory leading to the proposal of these growth exponent 29 makes assumptions about the underlying growth dynamics which break down in this region. This leaves us without an accurate theoretical estimate of the characteristic domain size at the crossover.

\section{F. Radial pair-correlation}

It has been observed that DPD simulations of an ideal gas (i.e. $\alpha=0$ ) using the Hoogerbrugge-Koelman scheme have unusual structure in the radialpair-correlation function.25 Since continuous-time DPD satisfies detailed balance 19 we expect the radial pair-correlation function to have a constant unit value. This suggests that a good finite-difference algorithm would not display this unusual structure.

In order to test each of the finite-difference methods under consideration, systems of 40000 identical particles were evolved $(\alpha=0)$ from initially random configurations until they reached equilibrium (roughly $t=50$ ), at which point the radial 
pair-correlation function was calculated. Simulations were performed for each of the finite-difference algorithms, using a timestep of $h=0.05$ for all methods except Ferrario I and II, and Gear-4 $(\lambda=1, q=1)$. These results are shown in Fig. 8. Fig. 9 shows the results of the simulations using the Ferrario methods and Gear-4 with $\lambda=1$ and $q=1$, for which we used $h=0.01$ since they are unstable at the larger timestep. For comparison, the Gear-3 $\left(\lambda=\frac{1}{2}, q=1\right)$, Groot-Warren $\left(\lambda=\frac{1}{2}\right)$, and Hoogerbrugge-Koelman methods were also tested with the smaller timestep $(h=0.01)$ and so are also shown in Fig. 9. From these figures we can see that none of the methods are significantly better than the Hoogerbrugge-Koelman scheme, and three are noticeably worse: Gear-3 $(\lambda=1, q=1)$, Gear-4 $\left(\lambda=\frac{1}{2}\right.$, $q=1)$, and Groot-Warren $(\lambda=1)$. Decreasing the timestep gives a marked improvement, so that at $h=0.01$ all the methods capture the expected unit radial pair-correlation function to a good approximation.

\section{CONCLUSIONS}

The most significant differenfe between the simulations reported in this paper and those performed previously 224 is the improved precision of the measured behavior. We believe this is largely due to changing the discrete-time algorithm from Hoogerbrugge-Koelman to Groot-Warren. Coupled with its superior performance in the less-rigorous tests, we recommend the Groot-Warren method with $\lambda=\frac{1}{2}$ and timestep $h \leq 0.1$ as most suitable for DPD simulations. The Gear-3 algorithm with

$\lambda=\frac{1}{2}$ and $q=1$ is the obvious second choice, as it gave temperature and pressure to nearly the same accuracy as the Groot-Warren method $\left(\lambda=\frac{1}{2}\right)$, and is stable to a timestep nearly as large. Our only concern in recommending this modified Gear algorithm is its poor energy conservation.

We suspect the main limitation to improving the existing algorithms is their inability to properly address the stochastic component of the forces; there is some literature on Brownian dynamics simulations 32 that may be useful in finding an even better algorithm. Compared with the solution of ordinary differential equations, the numerical solution of stochastic differential equations is a recent area of mathematics, and one in which much more research is needed.33

\section{ACKNOWLEDGMENTS}

We are grateful to Peter Bladon, Bruce Boghosian, Alan Bray, Mike Cates, Pep Español, Simon Jury, Colin Marsh, John Melrose, and Matt Segall for helpful discussions. We thank NATO for a grant which has supported this work in part, as has the EPSRC (U.K.) Grand Challenge in Colloidal Hydrodynamics for access to the Cray T3D at the Edinburgh Parallel Computing Centre. KEN gratefully acknowledges financial support from NSERC (Canada) and the ORS Awards Scheme (U.K.).

${ }^{1}$ P. J. Hoogerbrugge and J. M. V. A. Koelman, Europhys. Lett. 19, 155 (1992).

${ }^{2}$ R. L. Burden and J. D. Faires, Numerical Analysis, 5th Ed. (PWS Publishing, Boston, 1993). 
${ }^{3}$ C. W. Gear, Numerical Initial Value Problems in Ordinary Differential Equations (Prentice-Hall, Englewood Cliffs, NJ, 1971), Ch. 3, 9.

${ }^{4}$ W. H. Press, S. A. Teukolsky, W. T. Vetterling and B. P. Flannery, ed., Numerical Recipes in C: The Art of Scientific Computing, 2nd ed. (CUP, Cambridge, 1992), Ch. 2, 16.

${ }^{5}$ M. P. Allen and D. J. Tildesley, ed., Computer Simulation in Chemical Physics (Kluwer, Dordrecht, 1992), Ch. 1.2, 5.6.

${ }^{6}$ M. P. Allen and D. J. Tildesley, Computer Simulation of Liquids (Clarendon, Oxford, 1987), Ch. 3.2, 7.4.3, 9, E.

${ }^{7}$ H. J. C. Berendsen and W. F. van Gunsteren, "Practical Algorithms for Dynamic Simulations", in G. Ciccotti and W. G. Hoover, eds., Molecular Dynamics Simulation of Statistical Mechanical Systems (Soc. Italiana di Fisica, Bologna, 1986).

${ }^{8}$ R. D. Groot and P. B. Warren, J. Chem. Phys. 107, 4423 (1997).

${ }^{9}$ J. M. Haile, Molecular Dynamics Simulation: Elementary Methods (Wiley, New York, 1992), Ch. 4.

${ }^{10}$ R. W. Hockney and J. W. Eastwood, Computer Simulation Using Particles (McGrawHill, New York, 1981), Ch. 4, 11.2.2, 12.1.3.

${ }^{11}$ E. S. Boek, P. V. Coveney and H. N. W. Lekkerkerker, J. Phys. Condens. Mat. 8, 9509 (1996).

12 E. S. Boek, P. V. Coveney, H. N. W. Lekkerkerker and P. van der Schoot, Phys. Rev. E 55, 3124 (1997).

13 A. G. Schlijper, P. J. Hoogerbrugge, and C. W. Manke, J. Rheol. 39, 567 (1995).

${ }^{14}$ B. M. Boghosian and W. Taylor, J. Stat. Phys. 81, 295 (1995).

${ }^{15}$ D. d'Humières and P. Lallemand, Complex Syst. 1, 633 (1987).

${ }^{16}$ P. Español and P. Warren, Europhys. Lett. 30, 191 (1995).

${ }^{17}$ C. W. Gardiner, Handbook of Stochastic Methods (Springer Verlag, Berlin, 1983).

${ }^{18}$ H. Risken, The Fokker-Planck Equation (Springer Verlag, Berlin, 1989).

${ }^{19}$ P. Español, Phys. Rev. E 52, 1734 (1995).

${ }^{20}$ D. H. Rothman and J. M. Keller, J. Stat. Phys. 52, 1119 (1988).

${ }^{21}$ P. V. Coveney and P. Español, J. Phys. A: Math. Gen. 30, 779 (1997).

${ }^{22}$ K. E. Novik and P. V. Coveney. Int. J. Mod. Phys. C 8, 909 (1997).

${ }^{23}$ D. Frenkel and B. Smit, Understanding Molecular Simulation: From Algorithms to Applications (Academic, San Diego, 1996), Ch. 4.2.3, 4.3, 6.1, E.

${ }^{24}$ P. V. Coveney and K. E. Novik, Phys. Rev. E 54, 5134 (1996); also P. V. Coveney and K. E. Novik, Phys. Rev. E 55, 4831 (1997).

${ }^{25}$ C. A. Marsh, G. Backx, and M. H. Ernst, Phys. Rev. E 56, 1676 (1997).

${ }^{26}$ J. M. V. A. Koelman and P. J. Hoogerbrugge, Europhys. Lett. 21, 363 (1993).

27 Truncation error is the loss in accuracy from a finite-difference algorithm using perfect arithmetic, and is analogous to the error from using a truncated Taylor series to approximate a function of a single variable. Round-off error is the precision lost by the computer due to finite precision arithmetic and approximations to functions.

${ }^{28}$ We believe this is the first time this 3-value corrector has been suggested, although its derivation is obvious.

${ }^{29}$ A. J. Bray, Adv. Phys. 43, 357 (1994).

${ }^{30}$ A. N. Emerton, P. V. Coveney, and B. M. Boghosian, Phys. Rev. E 55, 708 (1997).

${ }^{31}$ W. R. Osborn, E. Orlandini, M. R. Swift, J. M. Yeomans, and J. R. Banavar, Phys. Rev. Lett. 75, 4031 (1995).

${ }^{32}$ W. F. van Gunsteren and H. J. C. Berendsen, Mol. Phys. 45, 637 (1982).

${ }^{33}$ P. E. Kloeden and E. Platen, Applications of Mathematics, Vol. 23: Numerical Solution of Stochastic Differential Equations (Springer Verlag, Berlin, 1992). 


\begin{tabular}{lccc}
\hline \hline Method & Median & Std. dev. & Exponent \\
\hline Ferrario I (Eq. (17)) & 1.142 & 0.085 & \\
Ferrario II (Eq. (18)) & 1.054 & 0.039 & -0.024 \\
Gear-3 (Eq. (30), $q=1)$ & & & 0.0016 \\
Gear-3 (Eq. (30), $q=2)$ & & & -0.0044 \\
Gear-4 (Eq. (31), $q=1)$ & & & 0.00017 \\
Gear-4 (Eq. (31), $q=2)$ & & 0.039 & \\
Groot-Warren (Eq. (19)) & 1.054 & 0.25 & -0.0044 \\
Hoogerbrugge-Koelman (Eq. (15)) & 1.107 & & \\
Runge-Kutta (Eq. (20)) & & & \\
\hline \hline
\end{tabular}

TABLE I. Simple harmonic oscillator energy relative to initial energy for an homogeneous fluid, with a timestep of one tenth the period. A given median and standard deviation indicate periodic variation; an exponent describes exponential growth or decay.

\begin{tabular}{lccc}
\hline \hline Method & Median & Std. dev. & Exponent \\
\hline Ferrario I (Eq. (17)) & 1.00050 & 0.00042 & \\
Ferrario II (Eq. (18)) & 1.00049 & 0.00035 & $-2.6 \times 10^{-6}$ \\
Gear-3 (Eq. (30), $q=1)$ & & & \\
Gear-3 (Eq. (30), $q=2)$ & 0.99951 & 0.00012 & \\
Gear-4 (Eq. (31),$q=1)$ & 1.00032 & 0.000012 & \\
Gear-4 (Eq. (31), $q=2)$ & 1.00033 & 0.000013 & \\
Groot-Warren (Eq. (19)) & 1.00049 & 0.00035 & $-4.4 \times 10^{-7}$ \\
Hoogerbrugge-Koelman (Eq. (15)) & 1.0020 & 0.022 & \\
Runge-Kutta (Eq. (20)) & & & \\
\hline \hline
\end{tabular}

TABLE II. Simple harmonic oscillator energy relative to initial energy for an homogeneous fluid, with a timestep of one hundredth the period. A given median and standard deviation indicate periodic variation; an exponent describes exponential decay.

\begin{tabular}{cc}
\hline \hline Model & \\
Parameter & Value \\
\hline$\alpha_{0}$ & 7.063 \\
$\alpha_{1}$ & 7.487 \\
$\gamma$ & 5.650 \\
$\sigma$ & 1.290 \\
$m_{i}$ & 1 \\
$r_{c}$ & 1.3 \\
$\rho$ & 4 \\
\hline \hline
\end{tabular}

TABLE III. DPD model parameters (see Section [I]). 
FIG. 1. Relative equilibrium temperature $\left(k_{B} T\right)$ vs. timestep $(h)$ for an homogeneous fluid. Data are shown for the various methods: - - Ferrario I, $--\times--$ Ferrario II, -- $\square$ - - Gear-3 $(\lambda=1, q=1), \cdots \square \ldots$ Gear-3 $\left(\lambda=\frac{1}{2}, q=1\right),-\cdot-\circ-\cdot-$ Gear-3 $(\lambda=1, q=2), \longrightarrow$ Gear-3 $\left(\lambda=\frac{1}{2}, q=2\right),--\Delta^{--}$Gear-4 $(\lambda=1, q=1)$, - - - $\mathbf{\Delta - - ~ G e a r - 4}\left(\lambda=\frac{1}{2}, q=1\right), \cdots \nabla \cdots$ Gear-4 $(\lambda=1, q=2),-\cdot-\nabla-\cdot-\operatorname{Gear}-4\left(\lambda=\frac{1}{2}\right.$, $q=2),-\checkmark$ - Groot-Warren $(\lambda=1),---$ Groot-Warren $\left(\lambda=\frac{1}{2}\right),---*--$ Hoogerbrugge-Koelman, $\cdots \star \cdots$ Runge-Kutta. The solid line drawn at unity is for reference.

FIG. 2. Relative equilibrium temperature $\left(k_{B} T\right)$ vs. timestep $(h)$ for an homogeneous fluid (detail). Data are shown for the various methods: $--\times--$ Ferrario II, - - $\square-$ - - Gear-3 $(\lambda=1, q=1), \cdots \square$ Gear-3 $\left(\lambda=\frac{1}{2}, q=1\right),--\Delta_{--}$Gear-4 $(\lambda=1, q=1)$, $--\boldsymbol{\Delta}--$ Gear-4 $\left(\lambda=\frac{1}{2}, q=1\right),-\diamond$ - Groot-Warren $(\lambda=1),--\downarrow--$ Groot-Warren $\left(\lambda=\frac{1}{2}\right)$. The solid line drawn at unity is for reference.

FIG. 3. Equilibrium pressure $(P)$ vs. timestep $(h)$ for an homogeneous fluid. Data are shown for the various methods: - - Ferrario I, $--\times--$ Ferrario II, - - $\square---$ Gear-3 $(\lambda=1, q=1), \cdots \square \ldots$ Gear-3 $\left(\lambda=\frac{1}{2}, q=1\right),-\cdot-\circ-\cdot-\operatorname{Gear}-3(\lambda=1, q=2)$, $\longrightarrow$ Gear-3 $\left(\lambda=\frac{1}{2}, q=2\right),--\Delta--$ Gear-4 $(\lambda=1, q=1),-\cdots \Delta--$ Gear-4 $\left(\lambda=\frac{1}{2}, q=1\right), \cdots \nabla \cdots$ Gear-4 $(\lambda=1, q=2),-\cdot-\nabla-\cdot-\operatorname{Gear}-4\left(\lambda=\frac{1}{2}\right.$, $q=2),-\diamond-$ Groot-Warren $(\lambda=1),--\downarrow_{-}$Groot-Warren $\left(\lambda=\frac{1}{2}\right),---*--$ Hoogerbrugge-Koelman, $\cdots \star \cdots$ Runge-Kutta.

FIG. 4. Equilibrium pressure $(P)$ vs. timestep $(h)$ for an homogeneous fluid (detail). Data are shown for the various methods: $\cdots \mathbf{\square} \cdot$ Gear-3 $\left(\lambda=\frac{1}{2}, q=1\right),-\cdot-{ }^{-} \cdot-\cdot$ Gear-3 $(\lambda=1, q=2), \longrightarrow$ Gear-3 $\left(\lambda=\frac{1}{2}, q=2\right), \cdots \nabla \cdots$ Gear-4 $(\lambda=1, q=2)$, $-\cdot-\boldsymbol{\nabla}-\cdot-$ Gear-4 $\left(\lambda=\frac{1}{2}, q=2\right),---$ Groot-Warren $\left(\lambda=\frac{1}{2}\right),-\cdots *--$ Hoogerbrugge-Koelman, $\cdots \star \cdots$ Runge-Kutta.

FIG. 5. Growth of characteristic domain size for a single symmetric quench (Groot-Warren $\lambda=\frac{1}{2}, h=0.1$ ). Solid lines are of slope $\frac{1}{2}$ and $\frac{2}{3}$.

FIG. 6. Pressure difference as a function of bubble size (Groot-Warren, $\lambda=\frac{1}{2}, h=0.1$ ).

FIG. 7. Absolute viscosity $(\eta)$ vs. shear rate for an homogeneous fluid. Symbols indicate simulation size: $+N=1600$, and $\times N=6400$.

FIG. 8. Radial pair correlation function $g(r)$ vs. distance $r$ for a DPD ideal gas $(\alpha=0)$, with timestep $h=0.05$. Data are shown for the various methods: - - $\square$ - - - Gear-3 $(\lambda=1$, $q=1), \cdots \mathbf{\square} \cdots$ Gear-3 $\left(\lambda=\frac{1}{2}, q=1\right),-\cdot-\circ-\cdot-$ Gear-3 $(\lambda=1, q=2),-\bullet$ Gear-3 $\left(\lambda=\frac{1}{2}, q=2\right),-\cdots--$ Gear-4 $\left(\lambda=\frac{1}{2}, q=1\right), \cdots \nabla \cdots$ Gear-4 $(\lambda=1, q=2)$, $-\cdot-\boldsymbol{\nabla}-\cdot-$ Gear-4 $\left(\lambda=\frac{1}{2}, q=2\right),-\diamond-$ Groot-Warren $(\lambda=1),--\downarrow--$ Groot-Warren $\left(\lambda=\frac{1}{2}\right),---*--$ Hoogerbrugge-Koelman, $\cdots \star \cdots$ Runge-Kutta. The solid line drawn at unity is for reference. 
FIG. 9. Radial pair correlation function $g(r)$ vs. distance $r$ for a DPD ideal gas $(\alpha=0)$, with timestep $h=0.01$. Data are shown for the various methods: - + - Ferrario I, $--\times--$ Ferrario II, $\cdots \square \ldots$ Gear-3 $\left(\lambda=\frac{1}{2}, q=1\right),--\Delta_{--} \operatorname{Gear}-4(\lambda=1, q=1)$, -- Groot-Warren $\left(\lambda=\frac{1}{2}\right),---*--$ Hoogerbrugge-Koelman. The solid line drawn at unity is for reference. 


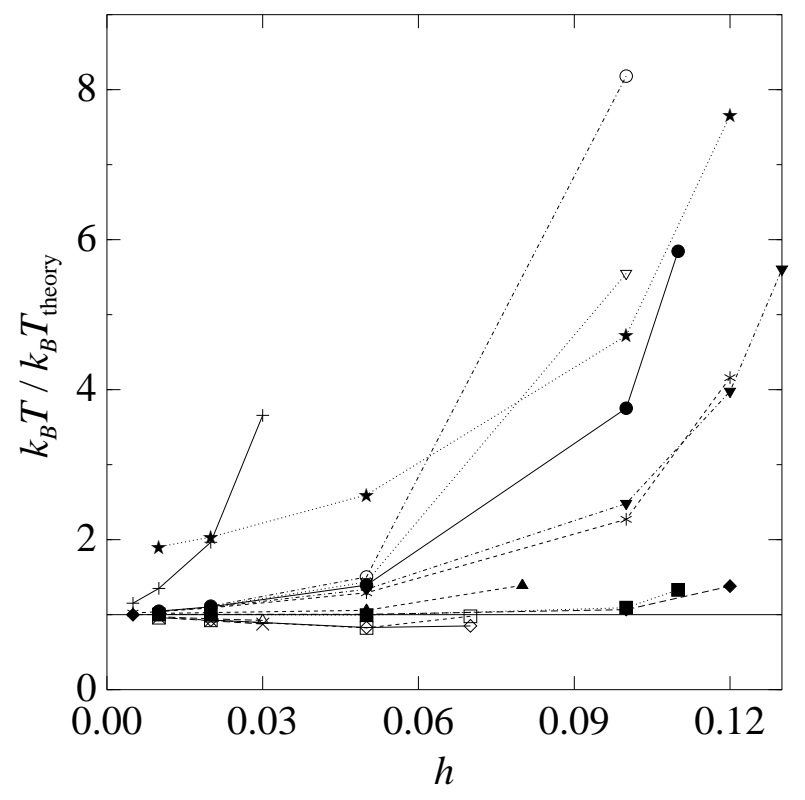

FIG. 1. Keir E. Novik, Journal of Chemical Physics 


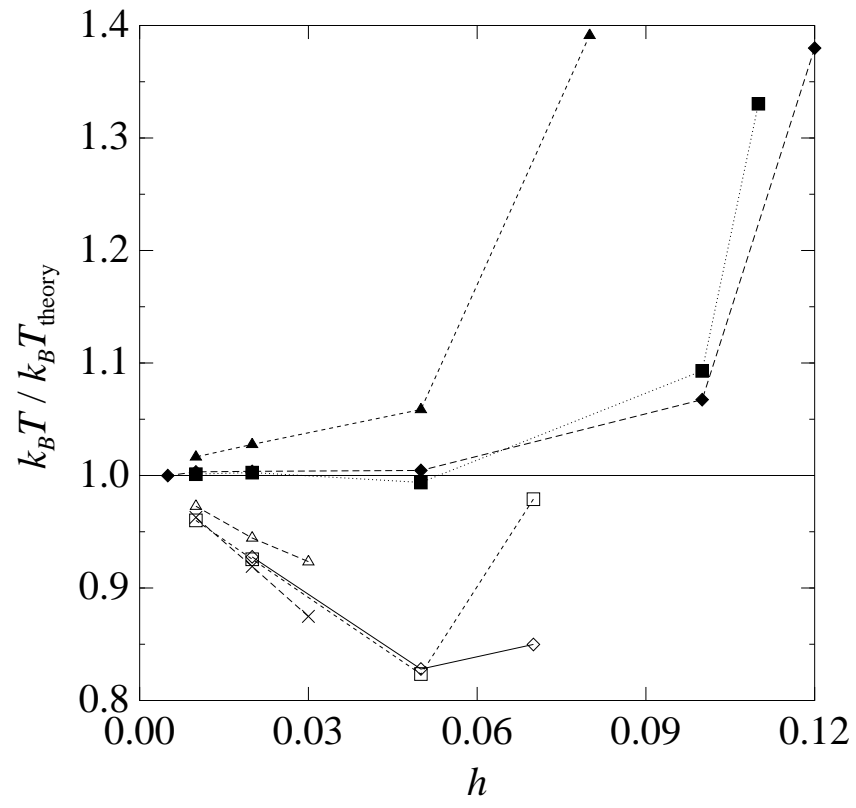

FIG. 2. Keir E. Novik, Journal of Chemical Physics 


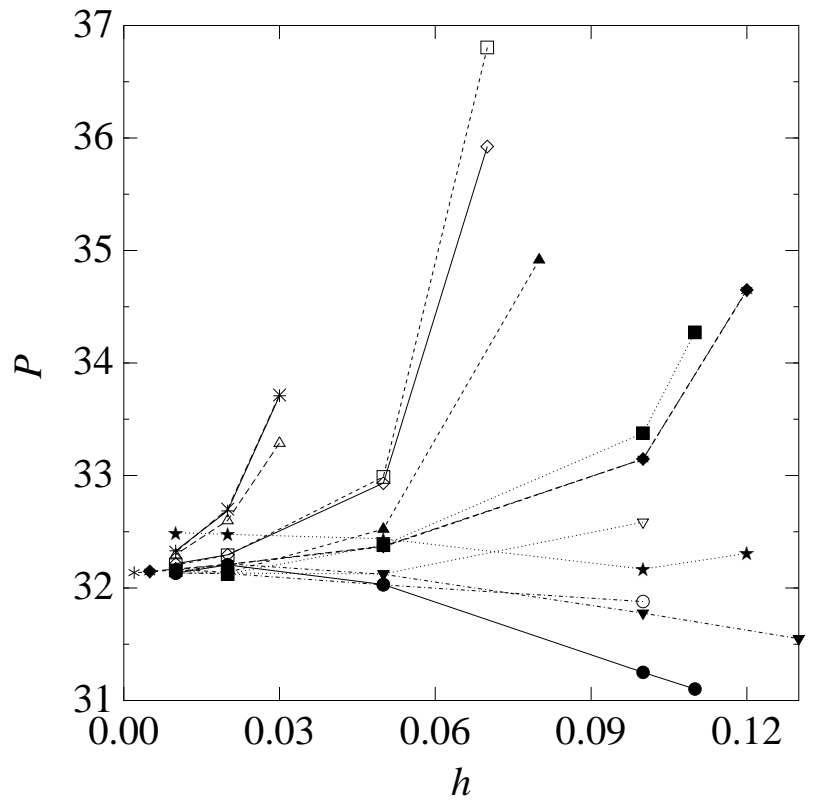

FIG. 3. Keir E. Novik, Journal of Chemical Physics 


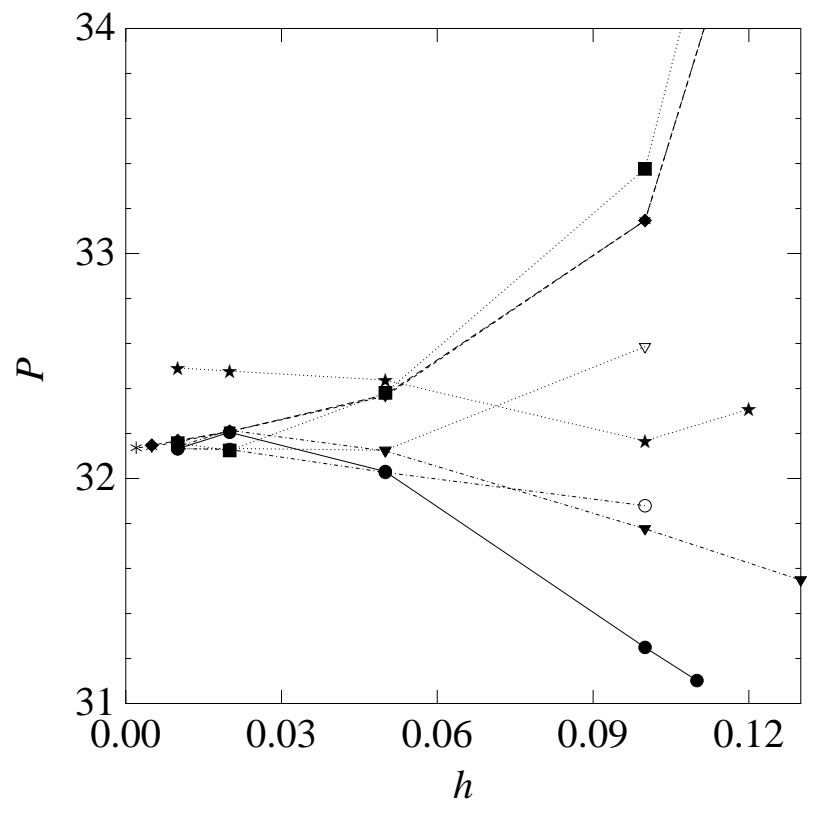

FIG. 4. Keir E. Novik, Journal of Chemical Physics 


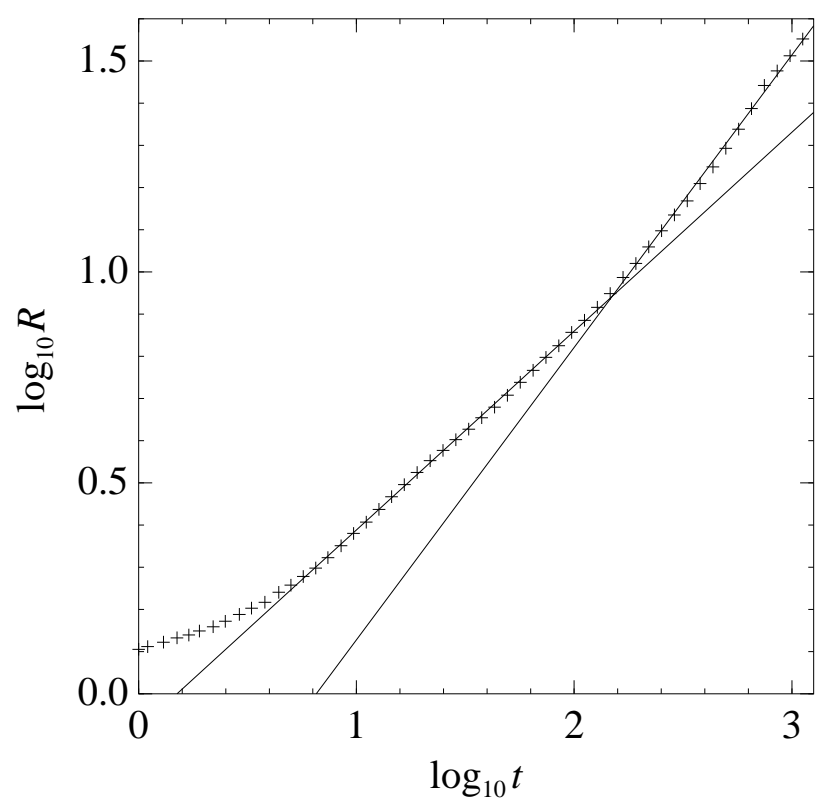

FIG. 5. Keir E. Novik, Journal of Chemical Physics 


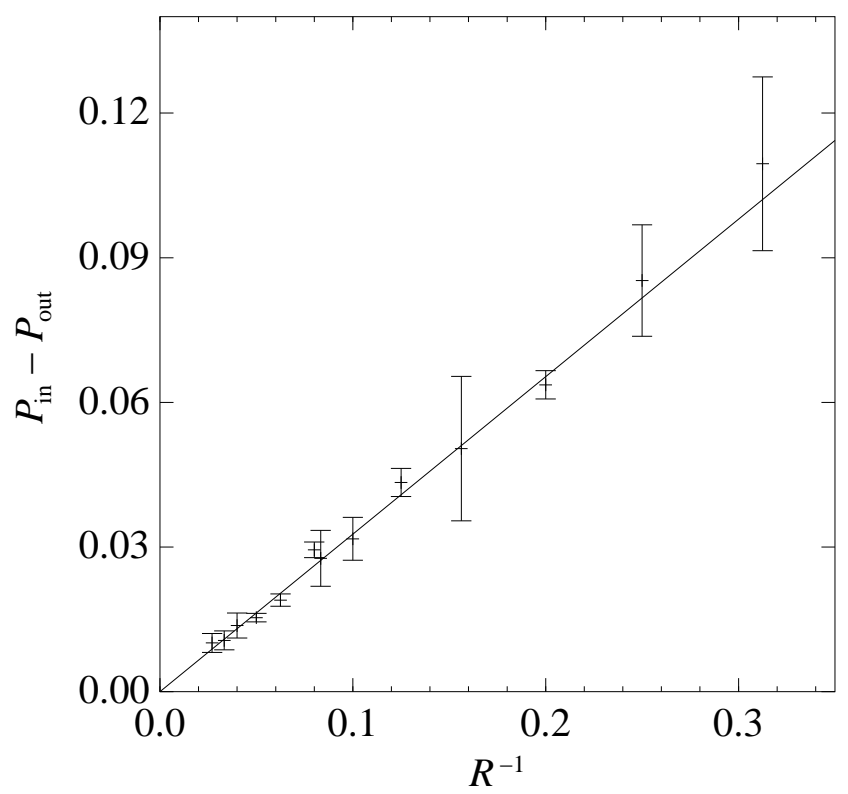

FIG. 6. Keir E. Novik, Journal of Chemical Physics 


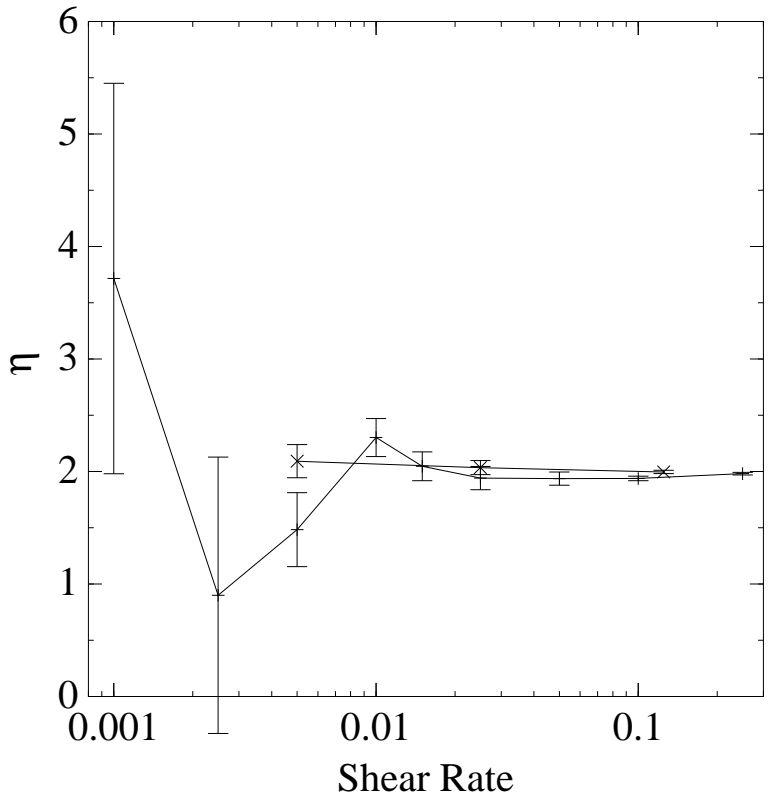

FIG. 7. Keir E. Novik, Journal of Chemical Physics 


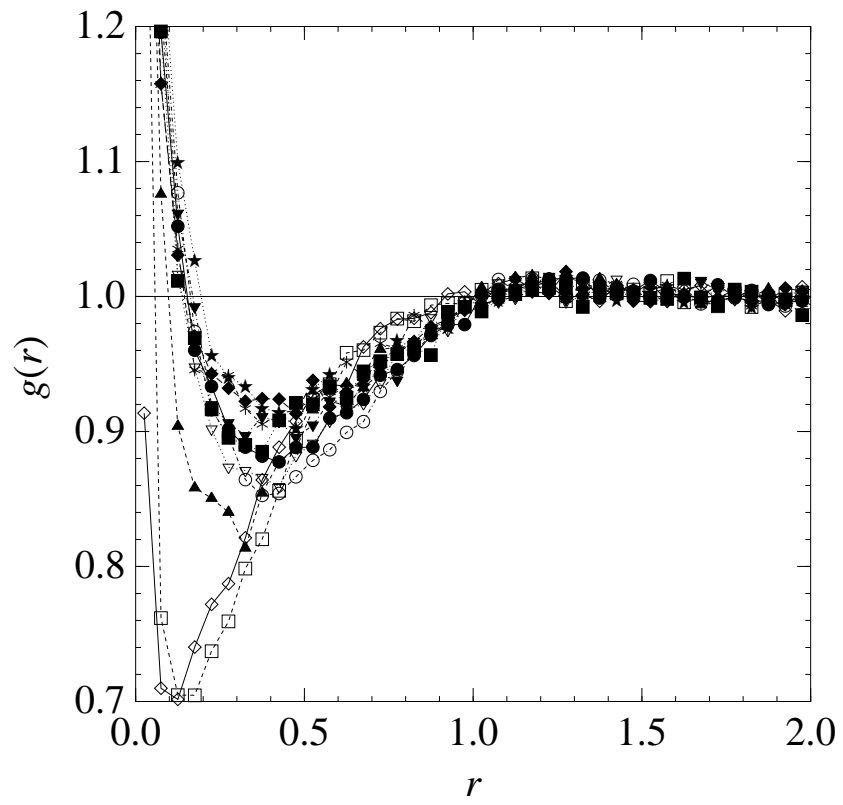

FIG. 8. Keir E. Novik, Journal of Chemical Physics 


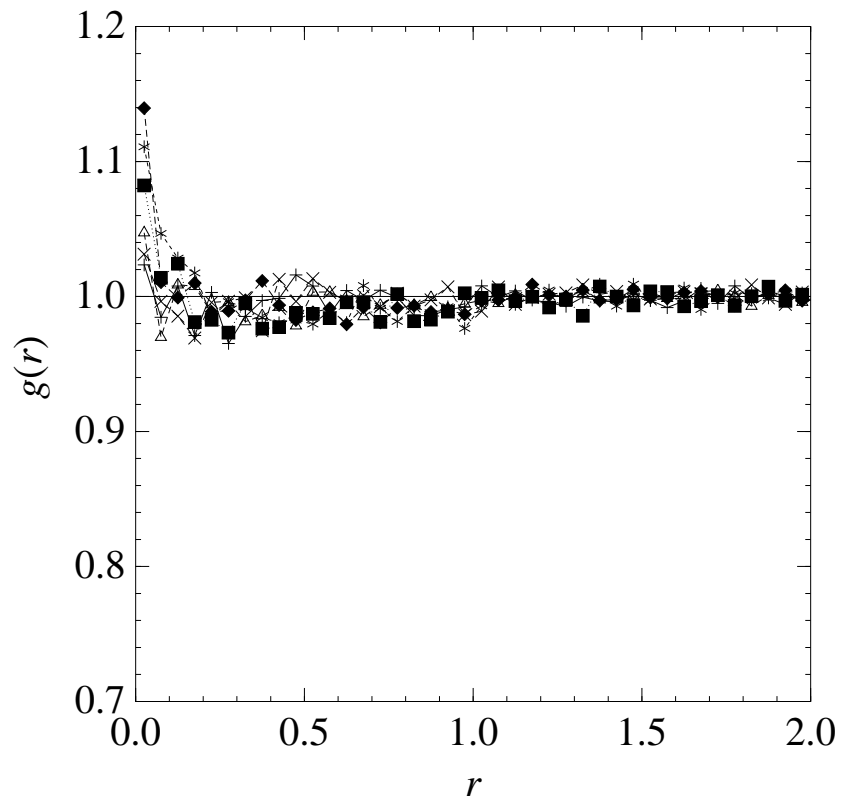

FIG. 9. Keir E. Novik, Journal of Chemical Physics 